\title{
BIONIC ARM: A PREVIEW FOR EXPANDING HORIZONS
}

\author{
Atharva Markale \\ Department of EEE \\ MIT- MAHE, Manipal, Karnataka, India
}

\begin{abstract}
Background- The increase in accidents destroys lives, especially if the person loses arm permanently. The amputees are not able to perform daily tasks on their own and thus they become dependent, lose self-esteem and confidence. The rapidly changing technology along with knowledge of medical science can address this issue of amputation and make lives of amputees better.
\end{abstract}

Methods:- This review literature discusses about development of bionic arm along with advances in engineering and medical sciences and summaries its working and future advancements.

Findings:- With progress in science, hook prosthetic was replaced by artificial prosthetic. They were acceptable and easily available but had lot of disadvantages. With progress in sensor technology, EMG (electromyogram) sensor is used to pick up signals from nerves of amputee and then drive the motors with help of microcontroller unit that will produce movements of bionic arm.

Conclusion:- The bionic arm has potential to change lives of amputees and so the medical and engineering people should research on making this version of bionic arm better in order to make it mimic the movements of natural arm.

Keywords - Electromyogram sensors, Artificial arm, artificial prosthetic, amputee.

\section{INTRODUCTION}

The human arm is a complex part. It has ability to generate force as per requirement, feel temperature vibration, can sense objects, the thumb enables to grip objects and move freely in all degree of freedom. Hence, loss of arm due to accidents can cause tremendous amount of problem to person.

The increase in number of accidents in changing world is a bane to society. Many times in accidents, people lose their arm and live a life of amputee thereafter. Amputation makes person dependent, also it degrades the self-esteem, confidence of person in society. To solve the issue various models of prosthetics were, introduced each were having drawbacks and were not able to mimic behavior of natural arm. To overcome this an electro mechanical system was used called bionic arm.
It is a mechanical arm controlled by nerve impulses, can act like an alternative to human arm.

Bionic arm is not yet popular due to its cost and availability but it has potential to change the lives of amputees.

\section{Proposed Algorithm}

Development of prosthetics

In order to help amputees the hook prosthetics were first started however its structure made it less useful and had social stigma.

Scientist carried of various experiments of arm replacement. The experiment had great success but the transplantation method has major drawbacks

Drawbacks of arm replacement technique

Replacement of arm restricts various natural movement of human arm. Its function gets reduced. The replacement surgery is costly, complicated and its rate of success remains as huge disadvantage.

\section{Alternative prosthetics}

Due to these drawbacks the old hook prosthetic and hand replacement technique was not widely used. The only practical solution that was feasible, was of making a replica of arm to provide structural support to amputee and allow them to perform few activities.

Development of prosthetics:- Current prosthetic are made up of carbon fiber like materials that are durable lightweight. These prosthetics were developed for providing structural support to amputee. It acts like a part of cosmetic, as most of fundamental movements are restricted.

\section{Drawbacks}

The artificial prosthetics does not allow person to move hand in all degrees of freedom. It restricts the motion of fingers making forearm remain as skeletal support, it does not allow user to produce force that is required for moving/pulling/pushing/lifting objects. Artificial arm lacks in getting sensory info that does not differentiate in temperature, vibrations, etc. Commonly available non carbon fiber prosthetic are bulky and have high weight that reduces patient acceptability. It causes low self-esteem as user is dependent on others for most of daily tasks Hence, to overcome all these 


\section{International Journal of Engineering Applied Sciences and Technology, 2020 \\ Vol. 5, Issue 1, ISSN No. 2455-2143, Pages 160-162 \\ Published Online May 2020 in IJEAST (http://www.ijeast.com)}

disadvantages with knowledge of medical and engineering sciences scientists innovators came with revolutionary concept 'bionic arm.'

Bionic arm:- Bionic arm is a electro mechanical device that picks up the signals from nerves of amputee amplifies it and convert in intuitive moments proportional to received impulses and via microcontroller it operates the servo motors that in turn moves the hand.

\section{Structure working and control}

\section{Electromyography}

It is method to capture electrical activity produced my skeletal muscles via sensor. Surface electromyography is usually used.

Human arm is a complex part that has lot of neurons and neuromuscular junctions. The accidents cause loss of the arm as whole or part. The neurons are still active in region from where the loss has took place and can be used to for getting electrical impulses required for driving bionic arm.

The signals derived from this method are of quite low power hence amplifiers are used to boost them and are fed to the control unit (here Arduino) as input. The Arduino Is complex device that includes a microcontroller memory few sensors and extra devices embedded on a single multiple purpose board

These electrical signals gets converted in digital form for processing by using complex algorithms. The electronic pattern recognition method is used to get certain movements.

According to necessities, the signal is sent to corresponding motor those which are responsible for circular, vertical, etc. movements. Further small variants of DC motor can be used in fingers for precise movements.

Possible techniques to improve the accuracy:- With help of machine learning the movements can be made accurate and natural. It is also possible to add force variation parameters to make the life of amputee simple.

Targeted motor reinnervation technique can be used to increase accuracy of myoelectronically controlled bionic arm. The targeted neuromuscles act as a natural amplifier and surface electromyograms can easily pick up accurate signals. There are techniques like implantation of bipolar differential EMG used to read intra muscular signals so that number of input sources increase making movements precise and natural.

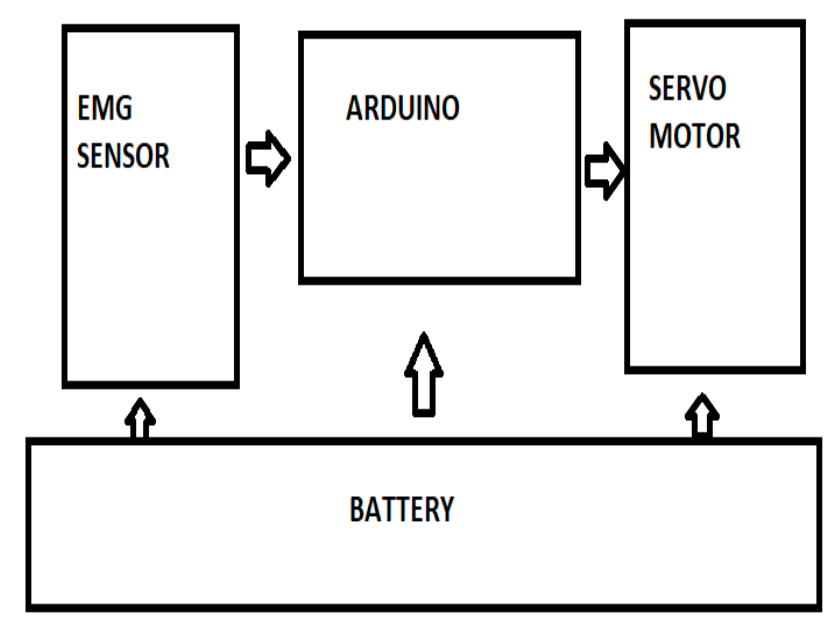

Fig.1 Block diagram of bionic arm

\section{Sensory system for bionic arm}

The human arm freely interacts with the surroundings and can sense the roughness temperature and amount of force required for object to push pull etc. This natural ability of human arm is complex and hard to replicate. The old prosthetics were not able to solve this issue. Broadly the sensory system can be categorized as information that user perceives and information that device collects.

Sensation using microphones:- There are few modern techniques used with help of sensors and control loops used for force detection control and production.

The mini ultrasonic microphones in hand will help in gripping activities and controlling the activities like gripping etc.

Direct interfacing is another method that directly involves interfacing of nervous system with electrodes of EMG for sensation. Advances in electrodes and algorithms will make the movements more natural by direct interfacing.

Few alternatives to this are multimodal plasticity where loss of one sensory input can be compensated by another.

The main benefit of adding sensory system is that user will be able to do movements grip objects and control forces making the work of bionic arm accurate and similar to natural arm.

\section{CONCLUSION}

The traditional prosthetic arm was just like a support provided for amputee. It lacked in many functions so was undesirable for patients also it added in decrease of self-esteem and made people dependent for working out daily choruses. The bionic arm is potential device that has addressed almost all issues of traditional prosthetics with electromechanical systems. Advances in EMG sensors, algorithms and applying machine learning to bionic arm will increase its potential to great extent and soon in future it may be able to replicate the function on 


\section{International Journal of Engineering Applied Sciences and Technology, 2020 \\ Vol. 5, Issue 1, ISSN No. 2455-2143, Pages 160-162 \\ Published Online May 2020 in IJEAST (http://www.ijeast.com)}

natural arm smoothly. Constant research in area of direct interfacing of central nervous system and EMG electrodes is slowly showing signs that bionic arm soon will be on par with natural arm and even it may outperform the human arm. With current developments in material science we have many new materials available which are durable, light in weight and affordable that will make it look aesthetic and more acceptable to patients. The battery management needs rework here. More efficient, economic and rechargeable batteries can be used in bionic arm. Advanced features for support and making it appear much better can be added later. Currently we are working with Arduino for controlling this arm, we can use alternative boards for it or exclusively design a FPGA for bionic arm. This area opens huge opportunity for engineers working in field of human machine interface, sensor and technology, material science and doctors

Cost and less awareness of bionic arm to patients is major drawback. Increase of advancements in biomedical field is showing positive sign and soon bionic arm may become an easily available affordable and acceptable in patients.

\section{REFERENCE}

[1] Clement, Rhys \& Bugler, Kate \& Oliver, Chris. (2011). Bionic prosthetic hands: A review of present technology and future aspirations. Journal of the Royal College of Surgeons of Edinburgh. 9. 336-40. 10.1016/j.surge.2011.06.001.

[2] Kulkarni, Tushar \& Uddanwadiker, Rashmi. (2016). Overview: Mechanism and Control of a Prosthetic Arm. Molecular \& cellular biomechanics: MCB. 12.

[3] Hargrove, Levi \& Simon, Ann \& Young, Aaron \& Lipschutz, Robert \& Finucane, Suzanne \& Smith, Douglas \& Kuiken, Todd. (2013). Robotic Leg Control with EMG Decoding in an Amputee with Nerve Transfers. The New England journal of medicine. 369. 1237-42. 10.1056/NEJMoa1300126.

[4] Mendes Júnior, José \& Janzen, F.C. \& Agostini Jr, Eloi \& Okida, Sergio \& Stevan Jr, Sergio. (2015). Design of an Electromyographic Signal Conditioning Circuit. IFMBE Proceedings. 49. 83-86. 10.1007/978-3-319-13117-7_22.

[5] Hutchinson, Douglas. (2014). The Quest for the Bionic Arm. The Journal of the American Academy of Orthopaedic Surgeons. 22. 346-351. 10.5435/JAAOS-22-06-346.

[6] Akazawa, Jun \& Yoshida, Masaki \& Okamoto, Takemasa \& Taniguchi, Kazuhiko. (2018). Electromyogram. 10.1007/978-3-319-69362-0_3.

[7] Coman, Ioan \& Dragulinescu, Ana \& Bucur, Doina \& Dragulinescu, Andrei \& Halunga, Simona \& Fratu, Octavian. (2019). Anthropomorphic EMG-Driven Prosthetic Hand. 10.1007/978-3-030-23976-3_1.
[8] Owen, Mahonri \& Fowke, Andrew \& Au, ChiKit. (2017). Development of a Dexterous Prosthetic Hand. Journal of Computing and Information Science in Engineering. 18. 10.1115/1.4038291.

[9] Seo, Minsang \& Kim, Hogyun \& Choi, Youngjin. (2017). Human mimetic forearm mechanism towards bionic arm. IEEE ... International Conference on Rehabilitation Robotics : [proceedings]. 2017. 1171-1176. 10.1109/ICORR.2017.8009408.

[10] Saint-Elme, Elina \& Larrier, Mervyn \& Kracinovich, Casey \& Renshaw, Dylan \& Troy, Karen \& Popovic, Marko. (2017). Design of a Biologically Accurate Prosthetic Hand. 12. 10.1109/WEROB.2017.8383866.

[11] K. Kiguchi, S. Kariya, K. Watanabe, K. Izumi, T. Fukuda, "An exoskeletal robot for human elbow motion support-sensor fusion adaptation and control", Systems Man and Cybernetics Part B: Cybernetics IEEE Transactions on, vol. 31, no. 3, pp. 353-361, 2001.

[12] O.A. Alsayegh, "EMG-based human-machine interface system", Multimedia and Expo 2000. ICME 2000. 2000 IEEE International Conference on, vol. 2, pp. 925-928 vol.2, 2000. 